\title{
Misidentification of Risk Group 3/Security Sensitive Biological Agents by MALDI-TOF MS in Canada: November 2015-October 2017
}

\author{
D Pomerleau-Normandin', M Heisz', M Su ${ }^{1 *}$
}

\begin{abstract}
Background: Matrix-Assisted Laser Desorption/lonization-Time of Flight Mass Spectrometry (MALDI-TOF MS) is a technology increasingly used in diagnostic identification of microorganisms. However, anecdotal evidence suggests that this technology is associated with misidentification of Risk Group 3 (RG3)/Security Sensitive Biological Agents (SSBA) resulting in exposure risks to laboratory personnel.
\end{abstract}

Objective: To investigate and characterize incidents related to the use of MALDI-TOF MS in Canada between November 6, 2015, and October 10, 2017.

Methods: Cases were identified from laboratory incident reports in the national Laboratory Incident Notification Canada (LINC) surveillance system. Eligible cases referred directly to MALDI-TOF MS or one of three RG3/SSBA organisms, Brucella species, Francisella tularensis and Burkholderia pseudomallei. A questionnaire was developed to identify potential risk factors leading to the exposure. Reporters from organizations with selected incidents were interviewed using the questionnaire. Data were entered into an Excel spreadsheet and standard descriptive statistical analysis performed to assess common characteristics and identify possible risk factors.

Results: There were eight eligible incidents and a total of 39 laboratory workers were exposed to RG3/SSBA organisms. In five (out of eight) of the incidents, the reporters indicated that their device was equipped with both clinical and research reference libraries. For six incidents where reporters knew the type of library used, only the clinical library was employed at the time of the incident even though both libraries were available in five of these incidents. In all eight cases, the exposure occurred during the sample preparation stage with analyses performed on an open bench and directly from the specimen. And in all eight cases, patient specimens were received without information regarding potential risk.

Conclusion: This first national study characterizing the nature and extent of laboratory incidents involving RG3/SSBA that are related to the use of MALDI-TOF MS identifies risk factors and provides baseline data that can inform mitigation strategies.

\section{Affiliation}

${ }^{1}$ Centre for Biosecurity, Public Health Agency of Canada, Ottawa, ON

*Correspondence: ming.su@ canada.ca

Suggested citation: Pomerleau-Normandin D, Heisz M, Su M. Misidentification of Risk

Group 3/Security Sensitive Biological Agents by MALDI-TOF MS in Canada: November 2015-October 2017.

Can Commun Dis Rep. 2018;44(5):100-15. https://doi.org/10.14745/ccdr.v44i05a04

Key words: MALDI-TOF MS, misdiagnosis, Risk Group 3 organisms, SSBAs

\section{Introduction}

The Matrix-Assisted Laser Desorption/lonization-Time of Flight Mass Spectrometry (MALDI-TOF MS) technology has been described as "a revolution in clinical microbial identification" (1). Identification of microorganisms in cell cultures can take up to 18 hours to complete; with MALDI-TOF this takes approximately 15 minutes (2). This new technology is increasingly used for routine microbe identification in both clinical and reference laboratories due to its simplicity, rapidity and high throughput capacity (3). This technology enables early diagnosis; the cost of the analysis is also greatly reduced (2).

MALDI-TOF mass spectrometry generates a characteristic spectrum, called a peptide mass fingerprint, formed as a result of the presence of up to 2,000 proteins found in a unique pattern in each organism (3). The MALDI-TOF MS software subsequently compares this pattern of proteins to an internal reference library that contains the spectra of known organisms. Because each 
bacterial species has a unique protein composition, the spectra enables accurate identification (4).

The main limitation of this technology is that the reference libraries must contain the spectrum of the organism in a sample to produce reliable identification (5). In the absence of the correct spectrum, the device will either not identify the organism or provide the identity of a similar organism. Close (but not exact) identifications can be a problem as closely related organisms can have different risk levels and therefore different safety protocols in the laboratory setting.

Organisms are classified in two ways: by risk group, based on an organism's pathogenicity, virulence, availability of treatment and the risk of spread to individuals or the public (Table 1); and as Security-Sensitive Biological Agents (SSBAs), a subset of human pathogens that pose a risk due to their potential for use as a biological weapon (6). Some, but not all Risk Group (RG)3, and most RG4 organisms are SSBAs.

\section{Table 1: Definition of risk groups and examples of} biological agents

\begin{tabular}{|l|l|l|}
\hline \multicolumn{1}{|c|}{$\begin{array}{c}\text { Risk } \\
\text { Group(RG) } \\
\text { level }\end{array}$} & \multicolumn{1}{|c|}{ Definition (6) } & \multicolumn{1}{c|}{$\begin{array}{c}\text { Examples of biological } \\
\text { agents }\end{array}$} \\
\hline RG1 & $\begin{array}{l}\text { Low individual risk } \\
\text { Low community risk }\end{array}$ & $\begin{array}{l}\text { Acholeplasma spp. } \\
\text { Achromatium axoliferum } \\
\text { Acidaminobacter spp. }\end{array}$ \\
\hline RG2 & $\begin{array}{l}\text { Moderate individual risk } \\
\text { Low community risk }\end{array}$ & $\begin{array}{l}\text { Burkholderia multivorans } \\
\text { Escherichia coli } \\
\text { Salmonella enterica spp. }\end{array}$ \\
\hline RG3 & High individual risk \\
& Low community risk & $\begin{array}{l}\text { Burkholderia pseudomallei } \\
\text { Brucella spp. } \\
\text { Francisella tularensis }\end{array}$ \\
\hline RG4 & High individual risk & Alkhumra virus \\
& High community risk & Nipah virus \\
\hline
\end{tabular}

Abbreviation: spp, species

If a specimen contains an RG3 organism but is misidentified as RG2, it may be handled on an open bench, rather than in a biosafety cabinet. Thus when misidentification occurs, laboratory technicians may not be appropriately protected and this can lead to exposure incidents.

A number of studies have reported that misidentification of organisms by MALDI-TOF MS appeared to have been caused by the incompleteness of the reference libraries used (2,7-26). Two types of reference libraries are currently in use in Canada: clinical libraries with spectra approved by Health Canada and research libraries, which contain a wider selection of spectra but are not cleared for clinical applications. Library extensions containing RG3 and SSBA spectra must be purchased separately. In certain cases, extensions are subject to extensive import regulations $(10,27,28)$, making their acquisition laborious. Library extensions are therefore not necessarily equally accessible to all laboratories.
The Public Health Agency of Canada (PHAC) has the capacity to assess this risk of misidentification from MALDI-TOF MS at the national level through the Laboratory Incident Notification Canada (LINC) surveillance program. This was set up under the Human Pathogens and Toxins Act, which came into full force in 2015 and which made the reporting of laboratory incidents to PHAC mandatory. By doing so, PHAC is able to develop national statistics on biosafety and biosecurity issues and detect emerging trends in close to real time.

The LINC first identified issues related to MALDI-TOF MS when five exposure incidents involving the use of this technology were reported over a nine month period. All five incidents involved RG3/SSBA organisms: Burkholderia pseudomallei, Francisella tularensis or Brucella species. They are the causative agents of melioidosis, tularemia and brucellosis, respectively. They present an increased risk for laboratory technicians as they are easily transmitted through aerosols and because their slow growth on standard culture media often delays the suspicion of an RG3/ SSBA $(13,29-31)$. Detection of these five incidents triggered an investigation to assess the nature and extent of this problem at the national level.

The objective of this study was to describe the exposure incidents related to MALDI-TOF MS use in Canada between November 2015 and October 2017, and to identify risk factors associated with these exposures.

\section{Methods}

\section{Eligible incidents}

Incidents between November 6, 2015 (date of the first incident) and October 10, 2017 (last date of data gathering) were reviewed. Reports were selected for further assessment based on the following inclusion criteria:

- Incidents, both exposure and non-exposure, that referred directly to MALDI-TOF MS; and

- Incidents, both exposure and non-exposure, involving Brucella species, F. tularensis or B. pseudomallei, for which the reports did not have enough details to confidently rule out the use of MALDI-TOF MS leading up to the incident.

An exposure incident was defined as "contact with, or close proximity to, infectious material or toxins that may result in infection or intoxication, respectively" (6). Non-exposure incidents were occurrences of inadvertent possession of an organism not authorized under the licence (i.e. possession of an RG3 in a containment level 2) (6).

Based on these criteria, 17 reports from 15 reporters (organizations) were selected for assessment.

\section{Investigation}

Two LINC program agents interviewed the reporters between October and November 2017, using a list of questions provided to the interviewee ahead of time. The questionnaire collected data on the possession of the MALDI-TOF MS device and its library extension(s), on the method of analysis, including whether analysis was performed on an open bench, on specific standard 
operating procedure (SOPs) for MALDI-TOF MS and triggers for enhanced SOPs and, in the opinion of the interviewee, whether the incident was caused, wholly or in part, as a result of misidentifying the organism. Data were also gathered on whether the medical staff, who requested the sample identification, provided information with the specimen (e.g., suspected diagnosis, patient medical and travel history) that may have alerted laboratory workers about the potential risk. An incident was confirmed as being part of the present study once the responsible reporter corroborated that the incident resulted from-or, at least, partly resulted from-misidentification or lack of identification from a MALDI-TOF MS device.

\section{Analysis}

Responses to the questionnaire were input into a spreadsheet designed for the purpose of this investigation. Excel 2010 was used for standard descriptive statistical analysis in order to assess and identify common characteristics.

\section{Results}

Of the 15 organizations contacted regarding 17 eligible incidents, 12 had a MALDI-TOF MS device. Among the remaining three organizations that did not have a device, two incidents still partly resulted from the use of MALDI-TOF MS. The reporters indicated that the specimens received for confirmation had previously been misidentified or not identified following MALDI-TOF MS analysis in a different laboratory.

The reporters at the 12 organizations that owned a MALDI-TOF MS device confirmed that, in six cases, the incidents were partly or wholly a result of relying on MALDI-TOF MS identification. With the addition of the two cases described above, in different laboratories, eight incidents related to misidentification or nonidentification by MALDI-TOF MS (Figure 1). These incidents represented over a third $(n=8 / 17)$ of all incident reports LINC received between November 6, 2015, and October 10, 2017, that involved the three selected biological agents.

Based on the information provided in the eight incident reports, of which two were non-exposure incidents, 39 laboratory technicians were exposed. Burkholderia pseudomallei was the biological agent involved in three of the incidents, Brucella species in another three, and F. tularensis in two (Table 2).

Table 2: Biological agent involved and number of individuals exposed per incident

\begin{tabular}{|c|l|c|}
\hline $\begin{array}{c}\text { Incident } \\
\text { number }\end{array}$ & \multicolumn{1}{|c|}{ Biological agent involved } & $\begin{array}{c}\text { Number of } \\
\text { exposed } \\
\text { individuals }\end{array}$ \\
\hline 1 & Burkholderia pseudomallei & 1 \\
\hline 2 & B. pseudomallei & 1 \\
\hline 3 & Francisella tularensis & 13 \\
\hline 4 & F. tularensis & 4 \\
\hline 5 & Brucella abortus & 15 \\
\hline 6 & B. pseudomallei & 5 \\
\hline 7 & Brucella melitensis & $\mathrm{n} / \mathrm{a}$ \\
\hline 8 & Brucella spp & $\mathrm{n} / \mathrm{a}$ \\
\hline
\end{tabular}

In five of the eight incidents the reporters indicated that their organization's MALDI-TOF MS device was equipped with both clinical and research reference libraries. In one incident, the device was only equipped with the clinical reference library, and in two incidents, the reporter did not know what type of reference library was used. However, in the five incidents where both clinical and reference libraries were available, all the analyses were performed using only the clinical reference library.

Figure 1: Case selection and eligible incidents

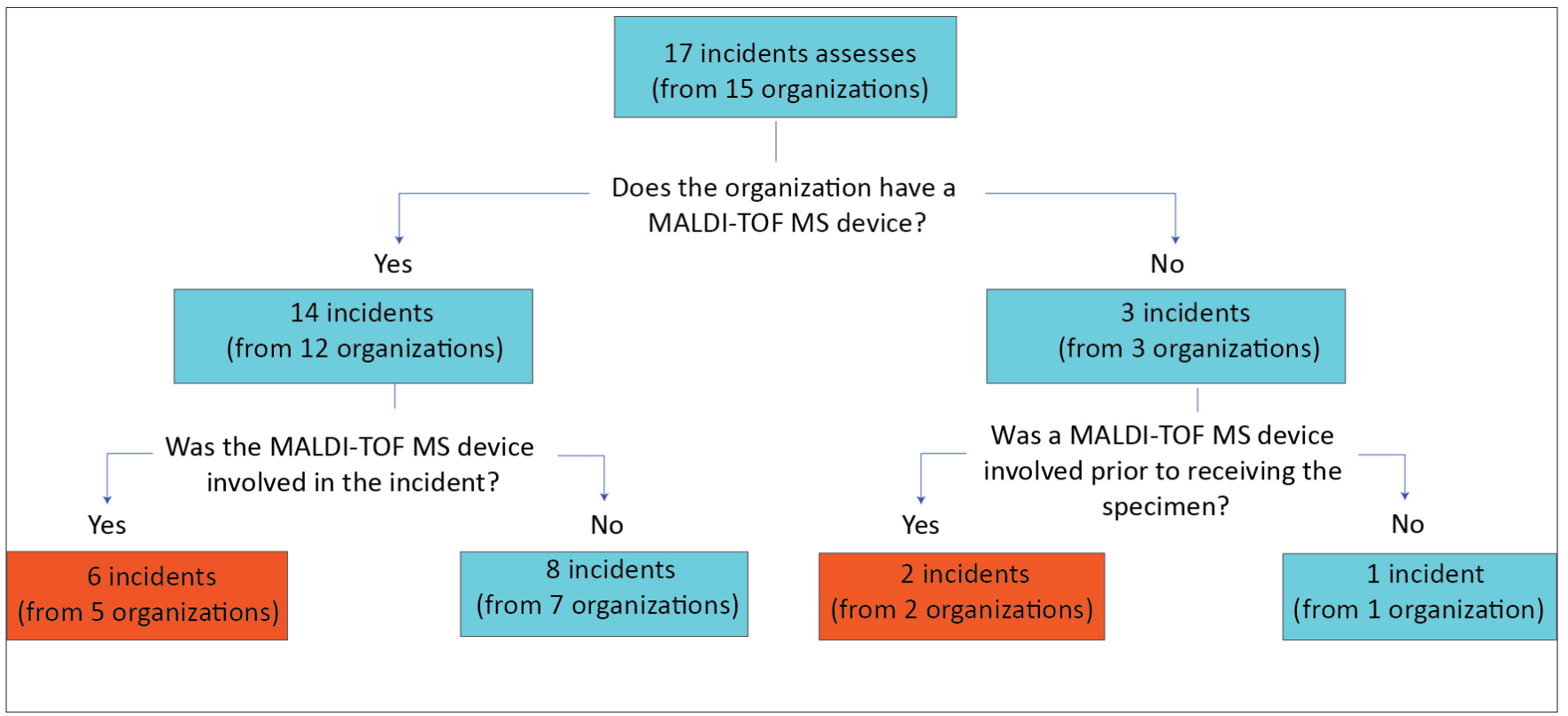

Abbreviation: MALDI-TOF MS; Matrix-Assisted Laser Desorption/lonization-Time of Flight Mass Spectrometry 
All eight incidents related to the use of MALDI-TOF MS occurred during the sample preparation stage, and all analyses were performed on an open bench, directly from the specimen. Furthermore, in every incident where the organization possessed a MALDI-TOF MS device $(n=6)$, the reporter developed enhanced SOPs designed specifically for the use of this technology following the incident. The triggers for these enhanced SOPs included a slow growth coupled with small gram-negative coccobacilli $(n=2)$, no identification results $(n=2)$, the geographic region from which the patient specimen originated $(n=1)$ and the specimen type $(n=1)$.

Finally, in all eight incidents related to the use of MALDI-TOF MS, specimens were received without clinical or travel history information (i.e. to trigger suspicion of RG3/SSBA agent).

\section{Discussion}

This is the first national study assessing laboratory incidents related to misidentification or lack of identification of RG3/SSBAs by MALDI-TOF MS devices. We found that 39 individuals were exposed to RG3/SSBA organisms in six exposure incidents involving MALDI-TOF MS. These organisms are consistent with other incidents reported in the literature $(3,10-12,15,19,22)$.

One risk factor associated with an exposure incident was reliance on the clinical reference library software. According to the reporters, although both clinical and research reference libraries were available in most cases, only the clinical libraries were in use when the incidents occurred as the two libraries cannot be used simultaneously and switching libraries requires a series of lengthy steps that are impractical under current workloads. Therefore, while complementary reference libraries such as research-use only or security-relevant libraries can help mitigate the risk of misidentification by relying on a wider selection of spectra, their utility remains limited as both types of library cannot be actively combined. These results are consistent with other reports in the literature that suggest MALDI-TOF MS clinical libraries do not reliably identify certain $R G 3 / S S B A s$ pathogens $(9,11,12,20,22)$.

A second risk factor associated with exposure incidents was an open-bench approach for sample preparation. All incidents occurred while preparing the sample and all analyses were performed directly from specimens on an open bench. This, however, does not actually reflect manufacturers' recommendations, which suggest that analysis be performed from extraction (32) that effectively deactivates pathogens in most cases and thereby reduces exposure risks.

A third risk factor associated with an exposure incident was the lack of alerting information on the laboratory requisition. A challenge with identifying these three diseases is that they usually present with nonspecific symptoms that can easily be mistaken for more common illnesses (8,25-27). As such, clinicians may not suspect an RG3/SSBA diagnosis and may not provide alerting information on the requisition forms (i.e. suspected diagnosis, patient travel history) that would cause the initiation of adequate safety measures for the manipulation of the patient specimens in laboratories $(30,33)$.
Our study identified two incidents from samples received following misidentification from a MALDI-TOF MS device in a different laboratory. This revealed that exposures due to a single specimen may occur in multiple laboratories.

\section{Strengths and limitations}

The main strength of this study is that it is based on mandatory, standardized and detailed reporting of laboratory incidents. Furthermore, it provides the baseline to assess the evolution of the problem over time.

There are also a few limitations to consider. The incident sample identified is likely incomplete for two reasons. First, the eligibility criteria may have missed some incidents. There is currently no efficient way to identify with certainty all incidents related to the use of MALDI-TOF MS among all laboratory incidents reported to LINC. Second, this investigation only captured incidents involving the three selected organisms. Indeed, MALDI-TOF MS devices have also been reported to misidentify or be unable to identify certain RG2 organisms as well (34). Finally, the small number of exposure incidents over almost a two-year time period is inherently unstable. Additional studies showing trends over time will further inform the extent of this problem.

\section{Conclusion}

This national surveillance study of laboratory incidents related to the misidentification of RG3/SSBAs by MALDI-TOF MS identified three risk factors: the reliance on clinical reference libraries; the use of an open-bench approach for sample preparation; and the lack of alerting information on the laboratory requisitions. This information can be used to raise awareness regarding the limitations of the MALDI-TOF MS technology and stimulate work on mitigation measures to help prevent similar incidents. It also provides the baseline for surveillance over time. The Centre for Biosecurity is continuing the investigation and working with stakeholders to address the issue and improve biosafety measures.

\section{Authors' statement}

DPN - Research, Investigation, Data analysis, Writing - Original Draft, Writing - Review and Editing MS - Investigation, Writing - Review \& Editing, Supervision $\mathrm{MH}-$ Writing - Editing, Supervision

\section{Conflict of interest}

None.

\section{Acknowledgements}

We would like to thank all the reporters contacted throughout the investigation process for their active collaboration. We would also like to extend our appreciation to all the licence holders and 
biological safety officers across Canada for providing high quality reports.

\section{Funding}

This work was supported by the Public Health Agency of Canada as part of its core mandate.

\section{References}

1. Calderaro A, Arcangeletti MC, Rodighiero I, Buttrini M, Gorrini C, Motta F, Germini D, Medici MC, Chezzi C, De Conto F. Matrix-assisted laser desorption/ionization time-offlight (MALDI-TOF) mass spectrometry applied to virus identification. Sci Rep 2014 Oct;4(6803):6803. https://dx.doi. org/10.1038\%2Fsrep06803. PubMed (https://www.ncbi.nlm. nih.gov/pmc/articles/PMC4213803/)

2. Ministère de la Santé et des Services Sociaux. Projet de réorganisation des laboratoires.Info Labo Bulletin. 2017 June;6(2). http://www.cisss-ca.gouv.qc.ca/fileadmin/ documents/Professionnels/Laboratoires/Info-Labo-2 Juin_2017.pdf

3. Singhal N, Kumar M, Kanaujia PK, Virdi JS. MALDI-TOF mass spectrometry: an emerging technology for microbial identification and diagnosis. Front Microbiol 2015 Aug;6(791):1-16. PubMed (https://www.ncbi.nlm.nih.gov/ pubmed/26300860?dopt=Abstract)

4. Clark AE, Kaleta EJ, Arora A, Wolk DM. Matrix-assisted laser desorption ionization-time of flight mass spectrometry: a fundamental shift in the routine practice of clinical microbiology. Clin Microbiol Rev 2013 Jul;26(3):547-603. http://dx.doi.org/10.1128/CMR.00072-12. PubMed (https:// www.ncbi.nlm.nih.gov/pubmed/23824373?dopt=Abstract)

5. Saraswathy N, Ramalingam P. Protein Identification by Peptide Mass Fingerprinting. In: Saraswathy N, Ramalingam P, editors. Concepts and Techniques in Genomics and Proteomics. 1th ed. Cambridge: Woodhead Publishing; 2011. p. 185-92.

6. Public Health Agency of Canada. Canadian Biosafety Standard. Second ed. Ottawa: Public Health Agency of Canada; 2015. https://www.canada.ca/en/public-health/services/canadianbiosafety-standards-guidelines/second-edition.html

7. Bizzini A, Greub G. Matrix-assisted laser desorption ionization time-of-flight mass spectrometry, a revolution in clinical microbial identification. Clin Microbiol Infect 2010 Nov;16(11):1614-9. http://dx.doi.org/10.1111/j.14690691.2010.03311.x. PubMed (https://www.ncbi.nlm.nih.gov/ pubmed/20636422?dopt=Abstract)

8. Canadian Agency for Drugs and Technologies in Health. MALDI-TOF Mass Spectrometry for Pathogen Identification: A Review of Accuracy and Clinical Effectiveness. Rapid Response Report. 2015 Oct 16:1-65. PubMed (https://www.ncbi.nlm.nih. gov/pubmedhealth/PMH0086127/)

9. Cunningham SA, Patel R. Importance of using Bruker's security-relevant library for Biotyper identification of Burkholderia pseudomallei, Brucella species, and Francisella tularensis. J Clin Microbiol 2013 May;51(5):1639-40. http:// dx.doi.org/10.1128/JCM.00267-13. PubMed (https://www. ncbi.nlm.nih.gov/pubmed/23447635?dopt=Abstract)
10. Dingle TC, Butler-Wu SM, Abbott AN. Accidental exposure to Burkholderia pseudomallei in the laboratory in the era of matrix-assisted laser desorption ionization-time of flight mass spectrometry. J Clin Microbiol 2014 Sep;52(9):3490-1. http:// dx.doi.org/10.1128/JCM.01238-14. PubMed (https://www. ncbi.nlm.nih.gov/pubmed/23447635?dopt=Abstract)

11. Jang HR, Lee CW, Ok SJ, Kim MJ, Bae MJ, Song S, Yi J, $\mathrm{Kim} \mathrm{KH}$. Melioidosis presenting as a mycotic aneurysm in a Korean patient, diagnosed by $16 \mathrm{~S}$ rRNA sequencing and matrix-assisted laser desorption/ionization time-of-flight mass spectrometry. Int J Infect Dis 2015 Sep;38:62-4. http://dx.doi. org/10.1016/j.ijid.2015.07.012. PubMed (https://www.ncbi. nlm.nih.gov/pubmed/26216763?dopt=Abstract)

12. Keller PM, Bruderer $V$, Müller F. Restricted Identification of Clinical Pathogens Categorized as Biothreats by MALDI-TOF Mass Spectrometry. J Clin Microbiol 2016;54(3):816. http:// dx.doi.org/10.1128/JCM.03250-15. PubMed (https://www. ncbi.nlm.nih.gov/pubmed/26763958?dopt=Abstract)

13. Kolecka A, Sanguinetti M, Boekhout T, Posteraro B. MALDI-TOF in Microbiology. First ed. Kostrzewa M and Schubert S, editors. Bremen: Caister Academic Press; 2016.

14. Lévesque S, Dufresne PJ, Soualhine H, Domingo MC, Bekal S, Lefebvre B, Tremblay C. A Side by Side Comparison of Bruker Biotyper and VITEK MS: Utility of MALDI-TOF MS Technology for Microorganism Identification in a Public Health Reference Laboratory. PLoS One 2015 Dec;10(12):e0144878. http:// dx.doi.org/10.1371/journal.pone.0144878. PubMed (https:// www.ncbi.nlm.nih.gov/pubmed/26658918?dopt=Abstract)

15. Lista F, Reubsaet FA, De Santis R, Parchen RR, de Jong $A L$, Kieboom J, van der Laaken AL, Voskamp-Visser IA, Fillo S, Jansen HJ, Van der Plas J, Paauw A. Reliable identification at the species level of Brucella isolates with MALDI-TOFMS. BMC Microbiol 2011 Dec;11:267. http://dx.doi. org/10.1186/1471-2180-11-267. PubMed (https://www.ncbi. nlm.nih.gov/pubmed/22192890?dopt=Abstract)

16. Mitchell PK, Campbell C, Montgomery MP, Paoline J, Wilbur C, Posivak-Khouly L, Garafalo K, Elrod M, Liu L, Weltman A. Notes from the Field: Travel-Associated Melioidosis and Resulting Laboratory Exposures - United States, 2016. MMWR Morb Mortal Wkly Rep 2017 Sep;66(37):1001-2. http://dx.doi. org/10.15585/mmwr.mm6637a8. PubMed (https://www.ncbi. nlm.nih.gov/pubmed/28934180?dopt=Abstract)

17. Saffert RT, Cunningham SA, Ihde SM, Jobe KE, Mandrekar J, Patel R. Comparison of Bruker Biotyper matrix-assisted laser desorption ionization-time of flight mass spectrometer to BD Phoenix automated microbiology system for identification of gram-negative bacilli. J Clin Microbiol 2011 Mar;49(3):887-92. http://dx.doi.org/10.1128/JCM.01890-10. PubMed (https:// www.ncbi.nlm.nih.gov/pubmed/21209160?dopt=Abstract)

18. Santos CR, Francisco E, Mazza M, Carolina A, Padovan B. Impact of MALDI-TOF MS in Clinical Mycology: Progress and Barriers in Diagnostics. In: Shah HN, Gharbia SE, editors. MALDI-TOF and Tandem MS for Clinical Microbiology. First ed. London: John Wiley and Sons Ltd; 2017. p. 211-30.

19. Suttisunhakul $V$, Pumpuang $A$, Ekchariyawat $P$, Wuthiekanun V, Elrod MG, Turner P, Currie BJ, Phetsouvanh R, Dance DA, Limmathurotsakul D, Peacock SJ, Chantratita N. Matrix-assisted laser desorption/ionization time-of-flight mass spectrometry for the identification of Burkholderia pseudomallei from Asia and Australia and differentiation between Burkholderia species. PLoS One 2017 Apr;12(4):e0175294. http://dx.doi. 
MALDI-TOF MS contribution to diagnosis of melioidosis in a nonendemic country in three French travellers. New Microbes New Infect 2016 Apr;12:31-4. http://dx.doi.org/10.1016/j. nmni.2016.04.004. PubMed (https://www.ncbi.nlm.nih.gov/ pubmed/27222715?dopt=Abstract)

27. Dual-use export controls. Brussels: European Commission; 2018 [cited 2018 Jan 20]. http://ec.europa.eu/trade/importand-export-rules/export-from-eu/dual-use-controls/

28. COUNCIL REGULATION (EC) Setting up a Community regime for the control of exports, transfer, brokering and transit of dual-use items, No 428/2009. http://eur-lex.europa.eu/legalcontent/EN/TXT/?qid=1489490204085\&uri=CELEX:0200 9R0428-20161116 (May 5, 2009)

29. Yagupsky P, Baron EJ. Laboratory exposures to brucellae and implications for bioterrorism. Emerg Infect Dis 2005 Aug;11(8):1180-5. http://dx.doi.org/10.3201/ eid1108.041197. PubMed (https://www.ncbi.nlm.nih.gov/ pubmed/16102304?dopt=Abstract)

30. Pedati C, House J, Hancock-Allen J, Colton L, Bryan K, Ortbahn D, Kightlinger L, Kugeler K, Petersen J, Mead P, Safranek T, Buss B. Notes from the Field: Increase in Human Cases of Tularemia--Colorado, Nebraska, South Dakota, and Wyoming, January-September 2015. MMWR Morb Mortal Wkly Rep 2015 Dec;64(47):1317-8. http://dx.doi. org/10.15585/mmwr.mm6447a4. PubMed (https://www.ncbi. nlm.nih.gov/pubmed/26632662?dopt=Abstract)

31. Wagar E. Bioterrorism and the Role of the Clinical Microbiology Laboratory. Clin Microbiol Rev 2016 Jan;29(1):175-89. http://dx.doi.org/10.1128/ CMR.00033-15. PubMed (https://www.ncbi.nlm.nih.gov/ pubmed/26656673?dopt=Abstract)

32. Bruker Daltonics. Instructions for Use MALDI Biotarget 48. Bruker Daltonics; 2011 December. https://www.criver.com/ sites/default/files/resources/InstructionsforUsingMALDIBiotarg et48.pdf

33. Traxler RM, Lehman MW, Bosserman EA, Guerra MA, Smith TL. A literature review of laboratory-acquired brucellosis. J Clin Microbiol 2013 Sep;51(9):3055-62. http://dx.doi.org/10.1128/ JCM.00135-13. PubMed (https://www.ncbi.nlm.nih.gov/ pubmed/23824774?dopt=Abstract)

34. Bilecen K, Yaman G, Ciftci U, Laleli YR. Performances and Reliability of Bruker Microflex LT and VITEK MS MALDI-TOF Mass Spectrometry Systems for the Identification of Clinical Microorganisms. BioMed Res Int 2015;2015(516410):1-18. http://dx.doi.org/10.1155/2015/516410. PubMed (https:// www.ncbi.nlm.nih.gov/pubmed/26793718?dopt=Abstract)

26. Walewski $V$, Méchaï $F$, Billard-Pomares $T$, Juguet $W$, Jauréguy $F$, Picard B, Tandjaoui-Lambiotte Y, Carbonnelle E, Bouchaud O. 